\title{
DENSITY OF METEOROIDS AND THEIR MASS INFLUX ON
}

\section{THE EARTH}

\author{
PULAT B. BABADZHANOV
}

\author{
Institute of Astrophysics, Dushanbe 734042, Tajikistan
}

\begin{abstract}
According to the investigation technique it is customary to divide meteoroids into three groups : 1) micrometeorites, with masses between $10^{-13} \mathrm{~g}$ and $10^{-6} \mathrm{~g}$, and densities between 0.4 and $4 \mathrm{~g} / \mathrm{cm}^{3} ; 2$ ) meteorites, for which the chemical composition is studied in detail, and having densities from 2 to $8 \mathrm{~g} / \mathrm{cm}^{3}$; 3) meteoroids proper, with masses larger than $10^{-6} \mathrm{~g}$, which produce meteoric phenomena in the Earth's atmosphere detected by optical and radar means.

On the basis of available photographic and radar observations in Dushanbe the influx $M(m)$ of meteoroids with mass equal or greater than $m$ is determined as $\log M(m)=3.7-0.1 \cdot \log m$ in $\mathrm{kg}$ to the Earth per day. This formula is applicable to a mass range from $10^{-6} \mathrm{~g}$ to $10^{2} \mathrm{~g}$.

The phenomenon of meteoroid fragmentation in the atmosphere was observed repeatedly by means of different methods and especially using the photographic technique of instantaneous exposure. Among four principal forms of fragmentation, the quasi-continuous fragmentation, i.e. a gradual release of smallest fragments from the surface of a parent meteoroid and their subsequent evaporation, is most common. The analysis of photographic observations shows that about $30 \%$ of meteoroids display this type of fragmentation. According to the theory of quasicontinuous fragmentation and on the basis of lightcurves of meteors, the densities of 85 meteoroids have been determined, which vary in the range from 0.1 to $8 \mathrm{~g} / \mathrm{cm}^{3}$. Not only porous and crumbly meteoroids but more dense stony and stony-iron meteoroids are also the subjects of fragmentation as well.
\end{abstract}

\section{Introduction}

The smallest bodies moving in the interplanetary space around the Sun and having sizes larger than molecules and smaller than asteroids are called meteoroids. Meteoroids are the products of disintegration of larger bodies : comets and asteroids. Hence, the investigation of the orbits and physical characteristics of meteoroids is important not only for meteor astronomy but also for understanding the physical nature of cometary nuclei and asteroids and for the cosmogony of the Solar system.

Compared with other celestial bodies the range of meteoroidal masses is large - from $10^{-13} \mathrm{~g}$ to hundreds tons, i.e. more than 20 orders of magnitude. This creates certain difficulties in the interpretation of meteor observations carried out by different methods, because different techniques (for example photographic or radar) may detect meteors produced by particles whose masses differ no more than three orders of magnitude, only a small part of the whole range.

According to the detection technique it is customary to divide the meteoroids into three groups : micrometeorites, meteorites, and meteoroids. In this paper we give brief information on micrometeorites and meteorites and dwell on the results 
of the investigation of meteoroid densities according to photographic observations of bright meteors.

\section{1. MICROMETEORITES}

Micrometeorites or micrometeoroids are the smallest particles under consideration here. Because of their large values of the surface to mass ratio, entering the Earth's atmosphere they radiate away the heat generated by collisions with air atoms and molecules. Therefore such particles do not reach the fusion point and they decelerate and drift down to the surface. The sizes of these particles are about tens of microns and smaller. The upper limit of their mass is equal to about $10^{-6} \mathrm{~g}$ which corresponds to the lower mass limit of the particles producing in the atmosphere the meteors of $+12^{m}-+13^{m}$ registered by high sensitive meteor radar systems (Kascheev and Tkachuk 1980; Baggaley et al., 1992).

The main tools for micrometeoroid detection are special detectors aboard space vehicles, meteor traps on high-altitude rockets and balloons, analyses of microcraters on lunar specimens, particle collection from high-flying aircrafts and from glacial accumulations, observations of the zodiacal light and gegenschein. These methods allow us to estimate the flux density of meteoroids of different mass ranges and to investigate their physical and chemical properties (structure, density and composition).

An analysis of micrometeorites collected in the high atmosphere shows that according to their physical properties and chemical composition they are similar to carbonaceous chondrites. Thus, Sutton and Flynn (1989) estimated the density of 11 cosmic dust particles to be in the range from 0.4 to $2 \mathrm{~g} / \mathrm{cm}^{3}$. According to studies of micrometeorite craters on the lunar specimens it was concluded that the mean density of micrometeorites lies in the range from 2 to $4 \mathrm{~g} / \mathrm{cm}^{3}$, i.e. close to the density of stone meteorites (Lebedinets 1981).

\section{2. METEORITES}

The chemical and mineralogical composition of meteorites has been studied in detail. According to chemical composition the meteorites are divided into three groups : a) iron, having an average density of $7.7 \mathrm{~g} / \mathrm{cm}^{3}$; b) stony-iron, with a mean density of $5.5 \mathrm{~g} / \mathrm{cm}^{3} ; \mathrm{c}$ ) stony, mainly chondrites, with a mean density of 3.6 $\mathrm{g} / \mathrm{cm}^{3}$.

Of particular interest are carbonaceous chondrites, which differ from other meteorites by high contents of volatiles, are enriched in carbon (in the form of organic compounds), and contain water (up to $20 \%$ ), sulphur, and a number of other minerals. Many authors consider that, according to their composition, carbonaceous chondrites are very close to the original matter of the Solar system. The density of carbonaceous chondrites varies in the range from 2 to $3.5 \mathrm{~g} / \mathrm{cm}^{3}$.

\section{3. METEOROIDS}

Meteoroids are the particles entering the atmosphere from interplanetary space and producing meteoric phenomena, i.e., creating sufficient luminescence and ionization to be detected by optical and radar methods. Their mass ranges between $10^{-6} \mathrm{~g}$ and 
$10^{3} \mathrm{~g}$ and beyond. The flux density of meteoroids, i.e. the number of meteoroids colliding with the unit surface per unit of time, is one of the main parameters for the studies of the interplanetary meteor matter and may be determined from photographic and radar observations of meteors.

The integrated meteoroid mass distribution may be expressed as :

$$
N(m)=b m^{1-s},
$$

where $N(m)$ is the number of meteoroids with mass equal to or greater than $m$, which intersect a fixed area within a fixed time $t$, and $b$ and $s$ are parameters to be determined.

To estimate the incident flux density using radar observations, we selected data on sporadic meteors producing overdense trails of duration $T>0.1 \mathrm{~s}$ corresponding to masses greater than $m=1.4 \times 10^{-3} \mathrm{~g}$. Some 226,000 meteors of that type were recorded in Dushanbe during 6800 hours of observation.

From double-station photographic observations of Super-Schmidt faint meteors and of small-camera bright meteors, and from the above radar observations, the mass index is found to be $s=2.1$ for meteoroids with mass from $10^{-3} \mathrm{~g}$ to $10^{2} \mathrm{~g}$. The incident meteoroid flux density turns out to be (Babadzhanov et al. 1990)

$$
\log \Phi(m)=-13.7-1.1 \log m,
$$

where $m$ is in $g$, and $\Phi(m)$ in $m^{-2} s^{-1}(2 \pi s t e r)^{-1}$. The logarithm of the cumulative number of meteoroids with mass equal or greater than $m$ coming to the whole Earth per day may be determined as

$$
\log N(m)=2.6-1.1 \log m,
$$

where $m$ is in $\mathrm{kg}$.

If the incident flux density of meteoroids with mass equal or greater than $m$ is known, then the influx of meteor matter to the terrestrial surface per day is given by

$$
\log M(m)=3.7-0.1 \log m,
$$

where $M(m)$ and $m$ are in $\mathrm{kg}$. This formula is applicable in an interval of mass from $10^{-9}$ to $10^{-1} \mathrm{~kg}$ (Babadzhanov et al., 1990).

According to (4) at $m \geq 10^{-9} \mathrm{~kg}$ the influx of meteoric matter to the Earth is estimated to be 40 tons per day or about 13,000 tons per year.

Present estimates of the meteoroidal mass influx on Earth, including meteoroids with masses between $10^{-17} \mathrm{~kg}$ and $10^{-1} \mathrm{~kg}$, meteorites and crater-forming bodies (asteroids and comets) are given in the paper by Kyte and Wasson (1986), which shows that in the total influx of all interplanetary bodies to Earth, the portion of large bodies with masses of $10^{10} \mathrm{~kg}$ and more is substantial.

Ceplecha (1992) estimated the total influx to the Earth of all interplanetary bodies in the mass range between $10^{-21} \mathrm{~kg}$ and $10^{15} \mathrm{~kg}$ to be $1.7 \times 10^{8} \mathrm{~kg}$ per year for the entire Earth surface. Recently Hughes (1993) estimated the total annual influx of meteoroids to the Earth around $2.2 \times 10^{8} \mathrm{~kg}$. 
Lebedinets (1991) came to the conclusion that Draconid type meteors having anomalous heights compared with the meteor luminosity theory are produced by organic meteoroids similar to CHON-particles. He supposed that in the interplanetary space at the distance $R=1 A U$ from the Sun about half of all meteoroids and $90 \%$ of Draconids must be organic. If this is the case the influx of meteoroids into the Earth must be at least twice as larger as estimated. This conclusion needs further experimental examination.

It should be noted that meteoric phenomena at high altitude do not necessarily require an organic composition of meteoroids, but may be caused by the existence of other volatiles in meteoroids of usual composition. This is a subject of extensive research.

A new method of determination of the sporadic meteoroid flux density, which is based on the meteor ionization of the E-layer of the Earth's atmosphere at night time, was developed in Dushanbe (Kolmakov 1990). It was shown that the maximum electron concentration of the E-layer before sunrise equal to $2 \times 10^{3}$ $\mathrm{cm}^{-3}$, corresponding to a flux density of

$$
\lg \Phi^{\prime}=-13.4-1.1 \lg M\left[m^{-2} s^{-1}(2 \pi s t e r)^{-1}\right],
$$

in a good agreement with the relation (2) determined directly according to meteor observations.

This method was applied to estimate the meteoroidal flux density on Venus and Mars using space probes data on the ionospheres of these planets. The obtained ratio of flux densities of sporadic meteoroids of masses larger than $10^{-7} \mathrm{~g}$ on Venus, Earth and Mars is $4: 1: 10$. These results may be explained by the fact that Venus is located closer to the Sun, where intensive disintegration of cometary nuclei take place and the perihelia of meteoroid orbits are concentrated. Mars is close to the asteroid belt and moreover has two small satellites, which pollute intensively the circum-martian space due to their own impacts with meteoroids.

Using observational data on the heights of meteors and fireballs produced by the meteoroids in a wide mass range from $10^{-4} \mathrm{~g}$ to $10^{8} \mathrm{~g}$ Ceplecha (1977) concluded that according to their structure and composition the meteoroids form three main groups : 1) ordinary chondrites, with a mean density of $3.7 \mathrm{~g} / \mathrm{cm}^{3} ; 2$ ) carbonaceous chondrites, with a mean density of $2.1 \mathrm{~g} / \mathrm{cm}^{3} ; 3$ ) "cometary" material with a density from 0.2 to $1 \mathrm{~g} / \mathrm{cm}^{3}$. These conclusions are confirmed by the results of our investigations on meteoroid densities taking into account quasi-continuous fragmentation (see later).

\section{Fragmentation and density of meteoroids}

The composition and density of meteoroids has been the subject of a number investigations in past years (Jacchia et al. 1967; Verniani 1967, 1969). The opinion that meteoroids have low densities was widely spread till recently. For example, analyzing the heights and decelerations of meteors photographed by Super-Schmidt cameras Jacchia et al. (1967) came to the conclusion that they have a crumbly structure and low densities (equal on the average to $0.26 \mathrm{~g} / \mathrm{cm}^{3}$ ). The main shortcoming of this and other papers is that all their results are based on a theory and 
methods which are applicable only to single non-fragmenting meteoroids, what cannot be considered as satisfactory.

Fragmentation of meteoroids in the Earth's atmosphere has been recorded repeatedly by both visual and photographic observations of meteors. The results of photographic observations carried out using the method of instantaneous exposure $\left(5.6 \times 10^{-4} \mathrm{sec}\right.$ each) show this phenomenon the most strikingly and completely (Babadzhanov 1983; Babadzhanov and Kramer 1967). At present one can consider as established that fragmentation is a normal type of ablation for the majority of meteoroids producing meteors registered by photographic and radar methods. Levin (1961) showed that neglecting fragmentation in the interpretation of meteor observations may lead to erroneous results.

Analyzing the data on photographic observations of meteors Levin (1961) distinguished four main forms of meteoroidal fragmentation : 1) the decay of a meteoroid into comparably large non-fragmenting debris; 2) the progressive disintegration of the original meteoroid into fragments, which continue to crumble into smaller debris; 3) the instantaneous spray of a large number of small particles giving rise to meteor flares; 4) the quasi-continuous fragmentation : a gradual release of the smallest fragments from the surface of a parent meteoroid and their subsequent evaporation.

Photographic observations of meteors show that along the meteor trajectory the fragmentations of types 1) and 3) may occur more than once. It is quite probable that a meteoroid may undergo different combinations of these fragmentation forms in the atmosphere (Babadzhanov et al., 1989).

Among the different forms of meteoroidal disintegration in the atmosphere described above, the quasi-continuous fragmentation is of the greatest interest. Both meteor wakes and terminal blendings, as well as the results of laboratory experiments provide evidence in favour of this mechanism of meteoroid disintegration.

The most plausible mechanisms leading to this type of fragmentation may be (Lebedinets 1980) : 1) the blowing off of the melted meteoroid layer in the case of iron meteoroids; 2 ) the ejection of the heated surface owing to fast evaporation of more volatile admixtures; 3 ) the thermodestruction and blowing off of the surface layer; 4) the separation of solid grains, assuming a grainy structure of the body, under the aerodynamic pressure or due to the melting of glue between grains (Hawkes and Jones 1975).

Thus the mere fact of meteoroidal fragmentation in the Earth's atmosphere is not yet an indication of a crumbly structure of meteoroids. Comparatively large and dense stony and iron meteoroids undergo fragmentation, too.

Proceeding from the results of an analysis of possible physical mechanisms of quasi-continuous fragmentation of different types of meteoroids Lebedinets (1987) a priori distinguished the following types of meteoroids according to their densities and fragmentation energies (Table I). These data are generally used by the different methods of determination of meteoroid densities which account for quasi-continuous fragmentation.

From the data of Table I the dependence of fragmentation energy on density may be approximated as

$$
Q_{f} \times 10^{-10}=0.10+0.18 \delta_{0}
$$


TABLE I

Densities $\delta_{0}$ and fragmentation energy $Q_{f}$ for different types of meteoroids

\begin{tabular}{|l|c|c|}
\hline Meteoroid type & $\delta_{0}\left(\mathrm{~g} / \mathrm{cm}^{3}\right)$ & $Q_{f} \times 10^{-10} \mathrm{erg} / \mathrm{g}$ \\
\hline Iron & 7.7 & 1.3 \\
Stony-iron & 5.5 & $1.0-1.3$ \\
Ordinary chondrite & 3.5 & $0.7-1.3$ \\
Carbonaceous chondrite CII & 2.5 & $0.5-0.7$ \\
Carbonaceous chondrite CI & 2.0 & $0.3-0.5$ \\
Carbonaceous chondrite C0 & 2.0 & $0.15-0.25$ \\
Dust ball & 1.0 & $0.2-0.6$ \\
Draconids type & 1.0 & $0.1-0.2$ \\
& & \\
\hline
\end{tabular}

First estimations of meteoroidal densities taking into account quasi-continuous fragmentation were obtained by Novikov et al. (1984), but a shortcoming of their work is that the theoretical heights of fragmentation beginning and the termination of meteoric phenomena were identified by means of the photographed heights of meteor appearance and disappearance, and this cannot be considered as satisfactory.

Basic equations of the theory of meteoroid quasi-continuous fragmentation were used by Lebedinets (1987a, 1987b) for the analysis of observed decelerations of faint photographic meteors and estimation of meteoroid densities. Using a mathematical simulation of the observed deceleration of Super-schmidt meteors, Lebedinets (1987a, 1987b) estimated density and fragmentation energy of 190 meteoroids (92 sporadic and 98 shower) of mass from 0.1 to $1 \mathrm{~g}$ (Table II).

It was found that out of 92 sporadic meteors 57 (or $62 \%$ ) were produced by meteoroids similar to carbonaceous chondrites, 22 (or $24 \%$ ) by meteoroids similar to ordinary chondrites, 8 (or $9 \%$ ) by iron meteoroids, 4 by stony-iron meteoroids, and 3 by very crumbly bodies of "dust ball" type. In the case of shower meteors, meteoroids of carbonaceous chondrite type and ordinary chondrite type were found more often, namely $48 \%$ and $29 \%$, but the meteoroids of iron and of stony-iron types, and very crumbly bodies interspersed with free ice were found rarely, only $5 \%, 6 \%$ and $12 \%$, respectively (Table II).

The main shortcoming of Lebedinets $(1987 \mathrm{a}, 1987 \mathrm{~b})$ work is that when determining meteoroid densities the meteor light curves are not taken into consideration. The value and reliability of the obtained results would increase considerably if the same meteors were analyzed by different methods. 
TABLE II

Distribution of sporadic and shower meteoroids according to Lebedinets (1987)

\begin{tabular}{|c|c|c|c|c|c|c|c|}
\hline \multirow{2}{*}{ Group } & \multicolumn{7}{|c|}{ Types of meteoroids in \% } \\
\cline { 2 - 7 } & Iron & Iron-stone & Ordin.chondr. & CII & CI & C0-DB & Draconids \\
\hline $\begin{array}{c}\text { Sporadic } \\
\text { meteors (92) } \\
\begin{array}{c}\text { Shower } \\
\text { meteors (98) }\end{array}\end{array}$ & 5 & 4 & 22 & 21 & 41 & 3 & 0 \\
\hline
\end{tabular}

\section{Determination of meteoroid densities}

The light curves of meteors are indicative of meteoroid ablation processes during their flight in the atmosphere. Since 1964 photographic observations of meteors were carried out in Dushanbe using both usual and instantaneous methods. Some unique meteor photographs obtained in Dushanbe by the method of instantaneous exposure with exposure times of $5.6 \times 10^{-4} \mathrm{sec}$ show clearly the effects of different types of meteoroid fragmentation. The most common type is the quasi-continuous fragmentation forming short-lived meteor wakes of up to several tens of metres in length. Instantaneous images of such meteors have a drop-like shape. (Babadzhanov 1983; Babadzhanov and Kramer 1967).

A theory of meteoroid quasi-continuous fragmentation is well developed at present. The formulas describing the variation of meteor luminosity along its visible trajectory depending on atmospheric density and parameters of quasi-continuous fragmentation have been obtained. According to this theory (Novikov et al.., 1984; Babadzhanov et al., 1984, 1988) the common expression describing the meteor luminosity along its trajectory is :

$$
\begin{gathered}
I(\rho)=\frac{9 \tau_{\nu} M_{0}^{3} V_{0} \cos Z_{R}}{2 H\left(R_{0} R_{1}\right)^{3}} \times \\
\times\left\{F_{1}(\rho) \Theta(a-\rho)+R_{1}^{3} F_{2}(\rho) \Theta(\rho-a) \Theta(b-\rho)+\frac{1}{30} F_{3}(\rho) \Theta(\rho-b) \Theta\left(\rho_{e}-\rho\right)\right\}
\end{gathered}
$$

where

$$
\begin{gathered}
F_{1}(\rho)=\rho\left\{\frac{1}{3}\left(\rho_{e}-\rho\right)^{2}\left[R_{1}^{3}-(a-\rho)^{3}\right]-\frac{1}{2}\left(\rho_{e}-\rho\right)\left[R_{1}^{4}-(a-\rho)^{4}\right]+\frac{1}{5}\left[R_{1}^{5}-(a-\rho)^{5}\right]\right\} \\
F_{2}(\rho)=\rho\left\{\frac{1}{3}\left(\rho_{e}-\rho\right)^{2}-\frac{1}{2} R_{1}\left(\rho_{e}-\rho\right)+\frac{1}{5} R_{1}^{2}\right\}
\end{gathered}
$$




$$
\begin{gathered}
F_{3}(\rho)=\rho\left(\rho_{e}-\rho\right)^{5} \\
a=\rho_{e}-R_{0}, \quad b=\rho_{e}-R_{1}
\end{gathered}
$$

$I(\rho)$ is the meteor luminosity at the height where the atmospheric density is equal to $\rho ; \tau_{\nu}, M_{0}, V_{0}$ are the luminous efficiency, pre-atmospheric meteoroid mass and velocity, respectively; $H$ is the scale height; $Z_{R}$ the zenith distance of the meteor radiant; $\rho_{e}$ the atmospheric density at the end height of meteor phenomena :

$$
\rho_{e}=2 \rho_{m}+0,75 R_{1}+\sqrt{\rho_{m}^{2}-0,0375 R_{1}^{2}}
$$

deduced from a condition that the maximum of light curve is in the range $a \leq$ $\rho_{m} \leq b ; \rho_{m}$ is the atmospheric density at the height of maximum luminosity, and $\rho$ the atmospheric density in the arbitrary point of the meteor trajectory; $a$ is the atmospheric density at the height of complete evaporation of fragments released at the moment of fragmentation beginning, while $b$ is the atmospheric density at the height of the end of fragmentation; $R_{0}$ and $R_{1}$ are the parameters determining the quasi-continuous fragmentation :

$$
R_{0}=\frac{6 Q_{f} M_{0}^{1 / 3} \delta_{0}^{2 / 3} \cos Z_{R}}{\Lambda A H V_{0}^{2}}, \quad R_{1}=\frac{9.67\left(Q-Q_{f}\right)(r \delta) \cos Z_{R}}{\Lambda^{\prime} A^{\prime} H V_{0}^{2}}
$$

$Q_{f}$ being the specific energy of meteoroid fragmentation, while $Q=8 \times 10^{10} \mathrm{erg} / \mathrm{g}$ is the specific energy of heating and evaporation of meteoroidal matter; $\Lambda, A, \delta_{0}$ are the heat transfer coefficient, the shape factor and the meteoroid density, respectively; $\Lambda^{\prime}, A^{\prime}, \delta$ - the same values for the fragments; $r$ is the fragment radius; $\Theta(x)$ is the Heaviside function : $\Theta(x)=1$ at $x>0$ and $\Theta(x)=0$ at $x \leq 0$.

In the case $R_{1} \rightarrow 0$, that corresponds to very small fragments and to a condition $Q \simeq Q_{f}$, the equation (7) coincides with the conventional equation of meteor light curves :

$$
I=\frac{9 I_{\max }}{4} \frac{\rho}{\rho_{\max }}\left[1-\frac{\rho}{3 \rho_{\max }}\right]^{2}
$$

where

$$
I_{\max }=\frac{2 \tau_{\nu} M_{0} V_{0}^{3} \cos Z_{R}}{9 H}, \quad \rho_{\max }=\frac{2 Q M_{0}^{1 / 3} \delta_{0}^{2 / 3} \cos Z_{R}}{\Lambda A H V_{0}^{2}}
$$

Using the observed values of $M_{0}, V_{0}, Z_{R}$ and the light curves of meteors, by the method of successive approximations we determined the values of the parameters $R_{0}$ and $R_{1}$ of equation (7) for which the theoretical and observed meteor lightcurves are in the best agreement.

The meteoroid density and mass of fragments may be estimated from the derived values of $R_{0}$ and $R_{1}$ if the values of $\Lambda, \Lambda^{\prime}, A, A^{\prime}, Q_{f}$ and product $r \delta$ are known $a$ priori. Meteors detected in the Dushanbe photographs are bright and produced by meteoroids of mass greater than $0.01 \mathrm{~g}$. According to Lebedinets (1991), for large meteoroids of mass between $0.01 \mathrm{~g}$ and $10 \mathrm{~g}$ the heat transfer coefficient depends on the mass $M_{0}$ as

$$
\Lambda=\Lambda_{0}+\left(1-\Lambda_{0}\right) \exp \left(-k M_{0}\right)
$$


where $\Lambda_{0}=0.03, k=0.25 \mathrm{~g}^{-1}$. It is assumed that $A=1.5, A^{\prime}=1.21, H=6 \mathrm{~km}$, $\Lambda^{\prime}=1$. Simulations of the meteor lightcurves were carried out for meteoroid densities between $10^{-2}$ and $10 \mathrm{~g} / \mathrm{cm}^{3}$ and products $r \delta$ of fragments between $10^{-4}$ and $10^{-1} \mathrm{~g} / \mathrm{cm}^{2}$. We adopted as reliable the values of meteoroid densities for which the mean deviation of the theoretical lightcurve from the observed one was minimum.

The results of the determination of densities of meteoroids belonging to different streams are given in Table III. The analysis of results shows that meteor matter contains at least three types of meteoroids : 1) dense stony bodies of ordinary chondrites or iron meteorite types (49 meteoroids); 2 ) meteoroids of carbonaceous chondrites of C II and C I types (11 meteoroids) 3) meteoroids of the carbonaceous chondrites of $\mathrm{C} 0$ or "dust ball" types ( 25 meteoroids).

\section{TABLE III}

Meteoroid densities

\begin{tabular}{|c|c|c|c|c|c|c|c|c|c|}
\hline \multirow[t]{2}{*}{ Shower } & \multirow[t]{2}{*}{ Parent } & \multicolumn{6}{|c|}{$\begin{array}{l}\text { Number of meteoroids having } \\
\text { different densities }\left(\mathrm{g} / \mathrm{cm}^{3}\right)\end{array}$} & \multirow[t]{2}{*}{ All } & \multirow{2}{*}{$\begin{array}{r}\begin{array}{r}\text { Mean } \\
\text { density }\end{array} \\
\mathrm{g} / \mathrm{cm}^{3}\end{array}$} \\
\hline & & $<1$ & $1-2$ & $2-3$ & $3-4$ & $4-5$ & $5-8$ & & \\
\hline Perseids & P/Swift-Tuttle & 3 & 5 & 5 & 13 & 8 & 3 & 37 & 3.4 \\
\hline Leonids & $\mathrm{P} /$ Tempel-Tuttle & - & 4 & 2 & 1 & - & - & 7 & 2.0 \\
\hline Geminids & 3200 Phaeton & 1 & - & 1 & - & 1 & 2 & 5 & 4.2 \\
\hline$\kappa$ Cygnids & $?$ & - & - & 1 & - & - & 1 & 2 & 5.0 \\
\hline Quadrantids & P/Machholz & 1 & 1 & - & 1 & - & 1 & 4 & 3.3 \\
\hline$\delta$ Aquarids & P/Machholz & - & - & - & 2 & 2 & 3 & 7 & 5.4 \\
\hline Taurids & P/Encke & 1 & - & - & - & 3 & - & 4 & 3.7 \\
\hline Sporadic & & 4 & 5 & 2 & 4 & 2 & 2 & 19 & 2.4 \\
\hline & All & 10 & 15 & 11 & 21 & 16 & 12 & 85 & 3.3 \\
\hline
\end{tabular}

Small differences of the results of the determination of meteoroid densities presented in Table III with respect to previous results (Babadzhanov 1992) are due to the fact that here we took into account two different kinds of quasi-continuous fragmentation (QCF), namely slow and fast QCF (Babadzhanov et al., 1984), whereas before only the first form of QCF was considered.

Slow fragmentation means that the fragments released at the beginning of fragmentation evaporate before the parent meteoroid body breaks completely into fragments. Fast fragmentation means that the complete destruction of the parent meteoroid body takes place before the disappearance of the fragments released at the moment of the fragmentation onset.

From Table III it follows that the composition of meteoroids in each stream may be quite different, from carbonaceous chondrites of $\mathrm{C} 0$ type or "dust balls" to iron type. The small amount of observational evidence does not permit us to carry out 
a more detailed analysis at present. The main conclusions of the present research can be summarized here : 1 ) not only friable meteoroids but also dense ones are subjects of fragmentation; 2) in the majority of the cases the meteoroid densities are similar to the densities of meteorites of different types; 3 ) according to these results all three groups of meteoroids (namely micrometeorites, meteoroids and meteorites) on the whole are similar.

\section{References}

Babadzhanov, P.B. : 1983, in Asteroids, Comets, Meteors (C.-I.Lagerkvist, H. Rickman, Eds.), 439-445, Uppsala universitet, Reprocetralen HSC, Uppsala, Sweden.

Babadzhanov, P.B. : 1992, in Asteroids, Comets, Meteors (A.W. Harris, E. Bowell, Eds.), 23-26, L.P.I., Houston.

Babadzhanov, P.B., Bibarsov, R.Sh. and Kolmakov, V.M. : 1990, Mass distribution and the flux of sporadic meteoroids, 56, Sov. Geophys. Committee, Moscow.

Babadzhanov, P.B. and Kramer, E.N. : 1967, in Physics and Dynamics of Meteors (Kresák L., Millman P.M., Eds.), 128-142, Reidel-Dordrecht.

Babadzhanov, P.B., Novikov, G.G. and Konovalova, N.A. : 1989, Astron. Vestnik XXIII, 277-281.

Babadzhanov, P.B., Novikov, G.G., Lebedinets, V.N. and Blokhin, A.V. : 1984, Preprint of Physical Technology Institute, 919, 55, Leningrad.

Babadzhanov, P.B., Novikov, G.G., Lebedinets, V.N. and Blokhin, A.V. : 1988, Astronomicheskij Vestnik, XXVII, 71-78.

Baggaley, W.J., Steel, D.I. and Taylor, A.D. : 1992, in Asteroids, Comets, Meteors (A.W. Harris, E. Bowell, Eds.), 37-40, L.P.I., Houston.

Ceplecha, Z. : 1977, in Comets, Asteroids, Meteoroids. Interrelation, evolution and origin (A.H. Delsemme, Ed.), 143-152, The University of Toledo.

Ceplecha, Z. : 1992, Bull. of the Amer. Astron. Soc., 24, 952.

Hawkes, R.L. and Jones, J. : 1975, Mon. Not. Royal Astron. Soc., 173, 339-356.

Hughes, D.W. : 1993, in Workshop on the Activity of Distant Comets (W.F. Huebner, H.U. Keller, D. Jewitt, J. Klinger, R. West, Eds.), 83-99, Southwest Research Institute, San Antonio, Texas.

Jacchia, L.G., Verniani, F. and Briggs, R.E. : 1967, Smithson. Contr. Astrophys., 10, No 1, 1-139.

Kascheev, B.L. and Tkachuk, A.A. : 1980, Result of Radar Observations of Meteor Orbits to $+12^{m}, 232$, Sov. Geophys. Committee, Moscow.

Kete, F.T. and Wasson, J.T. : 1986, Science, 232, 1225-1229.

Lebedinets, V.N. : 1980, Dust in the high atmosphere and space. Meteors., 247, Gydrometeoizdat, Leningrad.

Lebedinets, V.N. : 1981, Aerosol in the upper atmosphere and interplanetary dust, 280, Gydrometeoizdat, Leningrad.

Lebedinets, V.N. : 1987a, Astron. Vestnik, XXI, 65-74.

Lebedinets, V.N. : 1987b, Astron. Vestnik, XXI, 262-271.

Lebedinets, V.N. : 1991, Astron. Vestnik, XXV, 200-207.

Levin, B.Yu. : 1961, Bull. Komissii po kometam i meteoram, No 6, 3-10.

Lindblad, B.A. : 1976, In Interplanetary Dust and Zodiacal Light (H.Elsasser, H.Fechtig, Eds.), 373-378, Springer Verlag, Berlin.

Novikov, G.G., Lebedinets, V.N. and Blokhin, A.V. : 1984, Pisma v Astron. Journal., X, 71-76.

Sutton, S.R. and Flynn, C.J. : 1989, Meteoritics, 24, 329-330.

Verniani, F. : 1967, Smithson. Contr. Astrophys., 10, 181-195.

Verniani, F. : 1969, Space Sci. Rev., 10, 230-261. 